\title{
Building a second brain in the bowel
}

\author{
Marina Avetisyan, ${ }^{1}$ Ellen Merrick Schill, ${ }^{1}$ and Robert O. Heuckeroth ${ }^{2}$ \\ 'Washington University School of Medicine, St. Louis, Missouri, USA. ${ }^{2}$ Children's Hospital of Philadelphia Research Institute and Perelman School of Medicine, University of Pennsylvania, \\ Philadelphia, Pennsylvania, USA.
}

\begin{abstract}
The enteric nervous system (ENS) is sometimes called the "second brain" because of the diversity of neuronal cell types and complex, integrated circuits that permit the ENS to autonomously regulate many processes in the bowel. Mechanisms supporting ENS development are intricate, with numerous proteins, small molecules, and nutrients that affect ENS morphogenesis and mature function. Damage to the ENS or developmental defects cause vomiting, abdominal pain, constipation, growth failure, and early death. Here, we review molecular mechanisms and cellular processes that govern ENS development, identify areas in which more investigation is needed, and discuss the clinical implications of new basic research.
\end{abstract}

\section{Introduction}

Survival requires elegantly integrated mechanisms that control bowel motility, secretion, and blood flow to permit fluid and nutrient absorption and support waste elimination. If control of bowel function required conscious thought, there might be little else that we could do in life. Fortunately, the enteric nervous system (ENS) controls most aspects of bowel function $(1,2)$. The ENS is a complex network of neurons and glia that resides in the myenteric and submucosal plexus of the bowel. The myenteric plexus, located between longitudinal and circular muscle, primarily controls muscle contraction and relaxation. The submucosal plexus, found between circular muscle and bowel mucosa, regulates fluid secretion and absorption, modulates blood flow, and responds to stimuli from epithelium and lumen to support bowel function.

In most people, the ENS works well, making it easy to forget that the bowel needs its own nervous system. However, ENS defects may underlie common problems such as irritable bowel syndrome (IBS) (3) and less common problems such as Hirschsprung disease (HSCR) $(4,5)$, chronic intestinal pseudoobstruction syndrome (CIPO) (6), or gastroparesis (7). HSCR is a life-threatening birth defect in which the ENS is completely missing from distal bowel. In CIPO or gastroparesis, the ENS or other bowel cells are defective, which leads to dysmotility, pain, and difficulty maintaining enteral nutrition. In IBS, altered bowel motility and sensory responses cause pain accompanied by diarrhea or constipation, but health is not otherwise affected. ENS defects also occur in Parkinson's disease (8), diabetes (9), and inflammatory bowel disease (IBD) (10), and recent data suggest that ENS damage might play an early etiologic role in $\operatorname{IBD}(11,12)$ and Parkinson's disease (13-15). Here, we focus on cellular and molecular mechanisms controlling ENS development, highlighting areas that require further investigation and potential clinical implications of new discoveries.

Authorship note: Marina Avetisyan and Ellen Merrick Schill contributed equally to this work.

Conflict of interest: The authors have declared that no conflict of interest exists. Reference information: J Clin Invest. 2015;125(3):899-907. doi:10.1172/JCI76307.

\section{ENS morphogenesis}

The ENS forms from enteric neural crest-derived cells (ENCDCs) that delaminate primarily from vagal neural tube, with smaller contributions from sacral and upper thoracic neural tube (2,16-18). Vagal ENCDCs migrate through paraxial mesoderm before entering the bowel, and then migrate in a rostral to caudal direction to colonize the entire fetal bowel (Figure 1A). Vigorous ENCDC proliferation during migration is important for full bowel colonization $(19,20)$. ENCDCs differentiate into at least 20 neuronal subtypes or enteric glia (21), form ganglia, extend neurites, and establish and refine functional neural circuits to control the bowel (ref. 22 and Figure 1B). A subset of ENCDCs undergoes radial migration either inward to form the submucosal plexus or out of the bowel to form ganglia in the pancreas. These complex processes require transcription factors, cell surface adhesion molecules, receptors, extracellular ligands, cytoskeletal rearrangements, and diverse intracellular signaling molecules and are summarized in excellent recent reviews $(1,2,16,23-25)$.

\section{Retinoids, RET, and bowel colonization}

Migrating ENCDCs are surrounded by regulatory molecules that guide development. One early critical interaction occurs as ENCDCs migrate through paraxial mesoderm before invading foregut (E8.5 in mice, E2.5-3 in quail) $(17,26)$. During this transition, ENCDCs begin to express the receptor tyrosine kinase RET in response to local retinoic acid (RA) synthesis by paraxial mesoderm (ref. 17 and Figure 2A). This is important because RET supports ENCDC survival, proliferation, and migration (2, 27-33), and homozygous Ret inactivation prevents ENCDCs from colonizing bowel distal to the stomach $(34,35)$. Exogenous RA can substitute for an otherwise essential paraxial mesoderm interaction in quail to induce RET (17). Mice deficient in retinaldehyde dehydrogenase 2 , an enzyme that makes RA, also fail to express Ret and have total intestinal aganglionosis (36).

RET is the signaling receptor for glial cell line-derived neurotrophic factor (GDNF), neurturin, artemin, and persephin (37), ligands that bind preferentially to the coreceptors GFRA1, GFRA2, GFRA3, and GFRA4, respectively (38). GFRA1 and RET are coexpressed by migrating ENCDCs, but GFRA1 is also produced by 

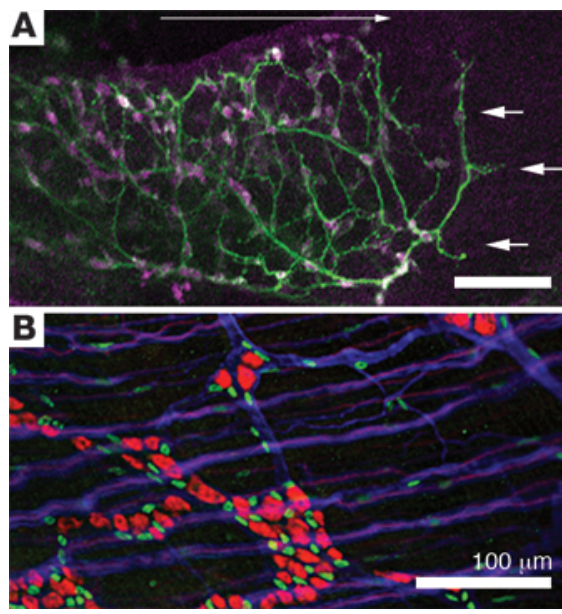

Figure 1. ENS morphogenesis. (A) Vagal ENCDCs migrate in a rostral to caudal direction through fetal bowel (long white arrow). At E12.5 ENS precursors have migrated halfway through the fetal colon. ANNA-1 antibody binds $\mathrm{HuC} / \mathrm{D}$ antigen and identifies enteric neurons (magenta), while TuJ1 binds neuron-specific $\beta$-III tubulin and labels neurites (green). ENCDCs migrate in chains though the bowel, but during the period of migration some precursors differentiate into neurons and extend neurites, including at the migration wavefront (white arrows). (B) Adult small bowel myenteric plexus, indicated by ANNA-1 antibody (red, neurons), SOX10 antibody (green, enteric glia), and TuJ1 antibody (blue), demonstrates clusters of neurons and glia in mature ganglia as well as many neurites within and between ganglia. Scale bars: 100 microns.

surrounding gut mesenchyme along with $\operatorname{GDNF}(39,40)$. Like RET, GDNF and GFRA1 are required for ENCDC survival, proliferation, and migration. Homozygous mutations in Ret, Gdnf, or Gfra1 cause indistinguishable ENS phenotypes in mice $(34,39,41-$ 44). RET mutations are also very common in people with HSCR $(2,16,23,45,46)$.

\section{Cell proliferation and bowel colonization}

One long-standing mystery is why ENCDCs colonize the entire fetal bowel, since individual ENCDCs appear to thrive anywhere along the bowel. The absence of essential intrinsic guidance cues for rostrocaudal migration is likely, as ENCDCs migrate in either direction through aganglionic bowel (47-49) and bypass a cecal zone with high concentrations of the chemoattractant GDNF (47). Theoretical modeling suggests that rostrocaudal bowel colonization is driven by ENCDC proliferation, which causes competition for space and trophic factor (ref. 19 and Figure 2B). In vitro and in ovo data support this idea, since reduced ENCDC proliferation or cell number may cause incomplete bowel colonization. For example, removal of vagal neural tube segments where ENCDCs originate causes distal intestinal aganglionosis $(50,51)$. RET mutations that reduce ENCDC proliferation or enhance cell death also cause bowel aganglionosis $(27,28)$, as does treatment with mycophenolic acid, an inhibitor of guanine nucleotide synthesis that reduces ENCDC proliferation but does not prevent ENCDCs from migrating (20). Finally, mutations in endothelin receptor B (EDNRB) or its ligand endothelin-3 (EDN3) cause premature differentiation and cell cycle exit (52-54), leading to HSCR in humans and distal bowel aganglionosis in mice $(55,56)$. Interestingly, Ednrb- and Edn3-deficient ENCDCs can colonize distal bowel normally if

accompanied by adequate numbers of WT ENCDCs, proving they are able to migrate into distal bowel $(57,58)$. One corollary to the hypothesis that competition for space drives ENCDC migration is that ENCDCs must be restricted to specific layers of the bowel. In part, sonic hedgehog from gut epithelium restricts ENCDCs to the outer bowel wall during migration (59), but the mechanism underlying this observation is not understood.

\section{Chain migration and cell adhesion}

Bowel colonization by ENCDCs is enhanced by chain migration, a process whereby ENCDCs preferentially contact each other while migrating (refs. 60, 61, and Figure 2C). The importance of ENCDC cell-cell contact is suggested by human L1 cell adhesion molecule (L1CAM) mutations that increase risk of HSCR, hydrocephalus, and corpus callosum defects (62-64). L1cam mutations also increase the number of isolated ENCDCs and reduce bowel colonization in mice (62). Ex vivo imaging shows that ENCDCs in chains migrate more quickly through bowel and with more directional persistence than do isolated ENCDCs (48). We do not, however, understand why contact among ENCDCs enhances bowel colonization. One hypothesis is that cells at the front of the migrating ENCDC chain pull trailing ENCDCs via L1CAMmediated adhesion. This seems unlikely, as migrating ENCDCs constantly change position, moving over each other $(47,48)$. Another possibility is that ENCDCs enhance bowel colonization by altering ECM to enhance migration (65) or by degrading ECM to create spaces through which to migrate. Consistent with this latter hypothesis, MMP2 inhibition slows ENCDC migration (66). Preferential ENCDC migration along neurites $(48,67)$ might also help cells navigate gut mesenchyme to preexisting gaps in the ECM. These hypotheses might explain why chain migration is more efficient than migration by isolated ENCDCs. Alternatively, isolated ENCDCs may simply remain where they are, since there is no competition for space or trophic factors to drive migration through the bowel. In either case, identifying mechanisms that support chain migration may provide new insight into human bowel motility disorders.

\section{Intracellular signaling and migration}

Complex intracellular signaling controls cytoskeletal rearrangements and focal adhesions essential for ENCDC migration. Mechanisms must be dynamically regulated because cells at the ENCDC migration wavefront behave differently from cells behind the wavefront. Recent, elegant studies of Förster resonance energy transfer have confirmed that PKA, RAC1, and CDC42 are differentially activated in migrating ENCDCs according to proximity to the wavefront (68). These proteins regulate the mode of cell migration $(69,70)$ and the efficiency of bowel colonization. Interestingly, both PKA inhibition and exogenous cAMP analogs slow ENCDC migration in organ culture $(68,71)$, but critical PKA targets in this context are not known. This suggests that localized PKA activation or moderate activation is needed to support migration. One possible PKA target is RET, which is phosphorylated and activated to enhance migration (71). RET in turn activates many molecules including RAC1 (72-74), which orchestrates cytoskeletal rearrangements to induce lamellipodia in cells migrating across 2D substrates (75). RAC inhibition slows ENCDC migration 
A
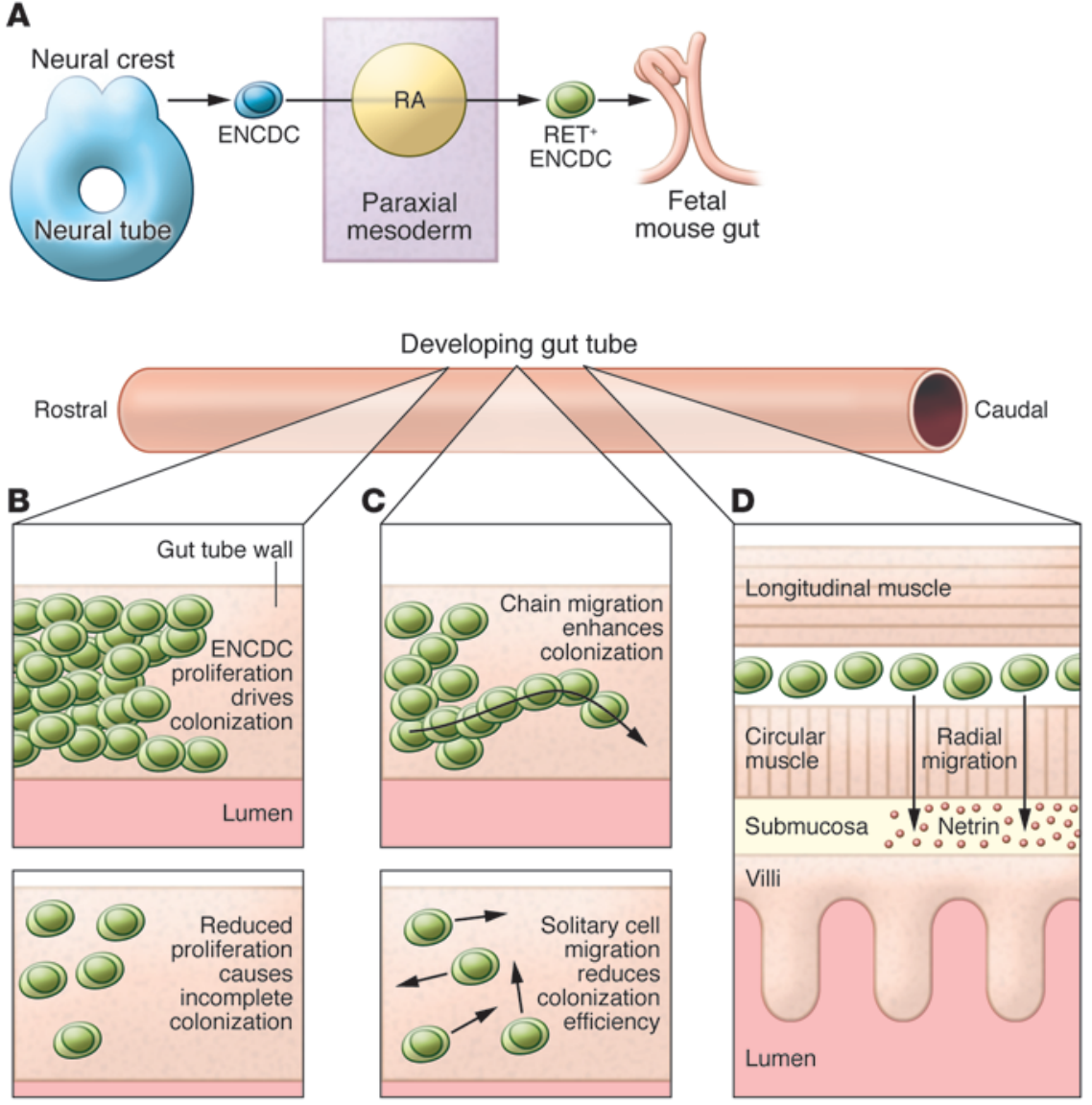

Figure 2. ENS development. (A) Murine vagal neural crest cells destined for the ENS delaminate from the neural tube at E8.5. These ENCDCs are exposed to RA as they migrate by paraxial mesoderm on their way to the foregut at E9. (B) Once ENCDCs are in developing bowel, efficient caudal migration relies on vigorous ENCDC proliferation (top panel), as disorders that reduce ENCDC proliferation (bottom panel) commonly cause incomplete bowel colonization. (C) Efficient ENCDC migration is facilitated by contact between migrating cells. Chain migration of ENCDCs is quicker and more directed than migration of isolated ENCDCs. Disorders that alter ENCDC cell adhesion also delay bowel colonization and may cause HSCR. (D) After ENCDCs have populated the whole developing bowel (E13.5 in mice) in the region of the future myenteric plexus, a subset of ENCDCs migrates inward radially to form the submucosal plexus. Radial migration is regulated by the RET-GDNF signaling axis and by netrin/ DCC chemoattraction. (E) nNOS-IR DG I neurons send caudal projections in the longitudinal axis, whereas CGRP-IR DG II neurons project circumferentially. Both DG I and DG II neurons are present at PO. However, only DGII neurons exhibit mature lamellar dendrites at this age, whereas most DC I dendrites are still filamentous. The proportion of DG I lamellar dendrites increases from P0 to P10. DC II projections do not grow in length from $P 0$ to P10, whereas DC I projections do, though their growth rate does not match that of the bowel. There is significant maturation of the ENS after birth, at least in rodents.
E Neuronal maturation

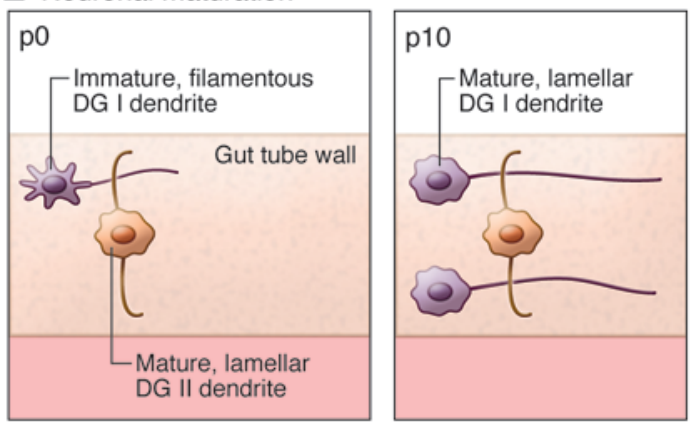

in vitro and in ex vivo organ culture $(68,73,76)$. Elevated RHOA, which often acts in opposition to RAC, also disrupts ENCDC chain migration and causes hypoganglionosis (77). Curiously, inhibition of ROCK, an effector of RHOA, enhances ENCDC migration in vitro on collagen but reduces ENCDC migration through bowel in organ culture (76). These apparently conflicting data suggest that in vitro results may not mimic mechanisms needed for migration through the bowel in vivo because different migration modes are optimal for different contexts. Evaluating mechanisms of ENCDC migration in natural, 3D environments such as bowel wall may therefore provide new insight into how medicines, toxins, genetics, or disease affect the developing ENS.

Additional complexity arises because signaling molecules may have different roles in different developmental contexts. For example, RA from paraxial mesoderm induces RET in neural crest cells migrating from the neural tube to the foregut (17). In contrast, when
ENCDCs have almost fully colonized the embryonic mouse bowel (E12.5), RET levels do not appear altered by RA receptor antagonists $(74,78)$. Instead, in distal bowel, RA reduces phosphatase and tensin homolog (PTEN) in ENCDCs at the migration wavefront to support migration. PTEN dephosphorylates phosphatidylinositol-3 phosphate (PIP3), a lipid that activates AKT and other proteins that, together with downstream effectors, orchestrate cytoskeletal rearrangements needed for migration and promote survival and proliferation (74). Interestingly, PTEN levels increase in ENCDCs behind the migration wavefront despite the presence of RA, and this increase in PTEN protein supports neuronal differentiation. RA also decreases SMURF1 ubiquitin ligase expression in axon tips of enteric neurons to reduce neurite growth (78). This appears to be a unique adaptation of developing enteric neurons that may facilitate migration. Thus, several RA-regulated genes play critical roles in ENS development, but effects of RA are context dependent. 


\section{Table 1. Neuronal subtype specification}

\begin{tabular}{|c|c|c|c|}
\hline Molecule & Experimental manipulation & Effect on enteric neuron subtypes & References \\
\hline GDNF & Overexpression in late development (E17) under glial GFAP promoter & Increased nNOS-IR neuron number; no change in ChAT neuron number & 32 \\
\hline \multirow[t]{2}{*}{ RET } & $\operatorname{Ret}^{-/-}$ & More dramatic loss of NOS neurons than other neurons in esophagus & 93 \\
\hline & Conditional loss of Ret plus rescue by $B c l-x L$ expression & Striking loss of NOS neurons in colon & 28 \\
\hline BMP & Overexpression of BMP4 under neuronal NSE promoter & Increased TrkC-IR, NT3-IR, nNOS-IR neurons & 90 \\
\hline \multirow[t]{2}{*}{ NT3 } & Loss of NT3 or the NT3 receptor, TrkC & $\begin{array}{c}\text { Decreased neuron number; preferential decrease in submucosal CGRP-IR } \\
\text { neurons }\end{array}$ & 131 \\
\hline & Overexpression of NT3 under dopaminergic DBH promoter & Increase in neuronal number & 131 \\
\hline EDN3 & Loss of the EDN3 or receptor, EDNRB & $\begin{array}{l}\text { Increased proportion of nNOS-IR and VIP-IR neurons; decreased proportion } \\
\text { of ChAT-IR neurons; region-specific changes in proportion of substance P-IR, } \\
\text { NPY-IR, galanin-IR, PACAP-IR and VIP-IR neurons }\end{array}$ & 132,133 \\
\hline ASCL1/MASH1 & Loss of Ascl1/Mash1 & $\begin{array}{c}\text { Decreased neuron number; reduced esophageal neuron number; loss of TC } \\
\text { neurons; loss of 5HT-IR neurons }\end{array}$ & 94,134 \\
\hline HAND2 & Loss of Hand2 & $\begin{array}{l}\text { Loss of or reduced nNOS/VIP-IR, reduced ChAT-IR, calretinin-IR, DAT-IR } \\
\text { neurons }\end{array}$ & 86,88 \\
\hline $5-\mathrm{HT}$ & Loss of neuronal 5-HT-synthesizing enzyme TPH2 & Decreased GABA-IR, DAT-IR neurons & 97 \\
\hline NET & Loss of NET & Decreased 5-HT-IR, calretinin-IR neurons & 98 \\
\hline
\end{tabular}

5-HT, 5-hydroxytryptamine; NT3, neurotrophin 3. Changes in expression levels for these proteins have been shown to alter the abundance of specific subtypes of enteric neurons in vivo.

Care is needed when evaluating protein function to consider the developmental context, since the role of many proteins (e.g., RET, BMP4, Notch, HAND2, Shh) changes depending on developmental stage, concentration, and cellular target $(2,16,23-25)$.

\section{ENS morphogenesis does not end when ENCDCs reach "The End"}

During rostrocaudal bowel colonization in mice, ENCDCs migrate in the location of the future myenteric plexus, an area rich in GDNF (79). Beginning at E14.5, some ENCDCs migrate inward (radial migration) to form the submucosal plexus, while at earlier stages other ENCDCs migrate into the pancreas to form ganglia near the Islets of Langerhans (refs. 23, 79, 80, and Figure 2D). Radial migration inward to the submucosal plexus and outward toward the pancreas is regulated by the chemoattractant netrin, which is secreted by fetal gut epithelium and pancreas and binds the receptor deleted in colon cancer (DCC), expressed by a subset of ENCDCs (79). Cells that move to the submucosal plexus also have less RET signaling than do adjacent ENCDCs (80). During radial migration, perimuscular gut mesenchyme downregulates Gdnf and mesenchyme closer to the lumen upregulates $G d n f(80)$. This change in GDNF localization provides additional stimulus for ENCDCs with low RET activity to migrate inward. How cells modulate levels of RET and how neighboring cells communicate to ensure that only a subset of cells leave the myenteric plexus is unknown.

As rostrocaudal migration nears completion, ENCDCs aggregate to become ganglia. Differential adhesion is important (81) and is mediated at least in part by neural cell adhesion molecule 1 (NCAM1) $(82,83)$. Polysialic acid (PSA) addition to NCAM1 in response to mesenchyme-derived BMP4 further promotes aggregation and reduces ENCDC migration. PSA-NCAM1 is not abundant in ENCDCs until gangliogenesis begins, and blocking the addition of PSA reduces ganglia formation (83). Some migratory ability is necessary for ganglia organization and may explain why mice lack- ing $\beta 1$ integrin, a receptor that binds ECM, have disorganized ganglia $(84,85)$. A more severe defect occurs in mice with conditional inactivation of the transcription factor Hand2 in ENCDCs, in which myenteric ganglia fail to form completely (86). Hand2 mutations reduce NCAM1, among many other defects. For example, HAND2 is needed for terminal differentiation of enteric neurons $(12,87,88)$ and this more global effect on neurogenesis may underlie the effect of Hand 2 mutations on ganglion formation and bowel motility. Additional work is needed to identify HAND2 targets and to define mechanisms controlling enteric ganglia formation.

\section{Neuronal subtype specification}

Enteric ganglia contain at least 20 distinct neuronal subtypes that differ in function, transmitters, neurite patterning, and electrophysiology (21). Undoubtedly diverse trophic factors, morphogens, and transcriptional regulators influence enteric neuron subtype specification (summarized in Table 1). However, mechanisms determining enteric neuron subtype are barely understood, and a genetic blueprint for specifying a single type of enteric neuron does not yet exist. Birth-dating studies show that progenitors for neuronal subtypes exit the cell cycle at different times throughout development (89-91). The observation that factors influencing ENCDC proliferation (e.g., GDNF) or differentiation (BMPs) may alter the ratio of enteric neuron subtypes within the bowel in a way that depends on neuronal birth date suggests a genetically programmed lineage commitment tied to the timing of cell cycle exit $(28,32,92,93)$. Cell fate, however, is determined by a combination of extrinsic signals from a cell's microenvironment and the intrinsic transcriptional programming that renders the cell competent to selectively respond to some extracellular signals. It is assumed that over time pluripotency of enteric neural precursors becomes increasingly restricted, resulting in greater lineage commitment. Reconstructing lineage relationships among enteric neuron subtypes, however, or defining events that restrict cell lineage is challenging with currently available 


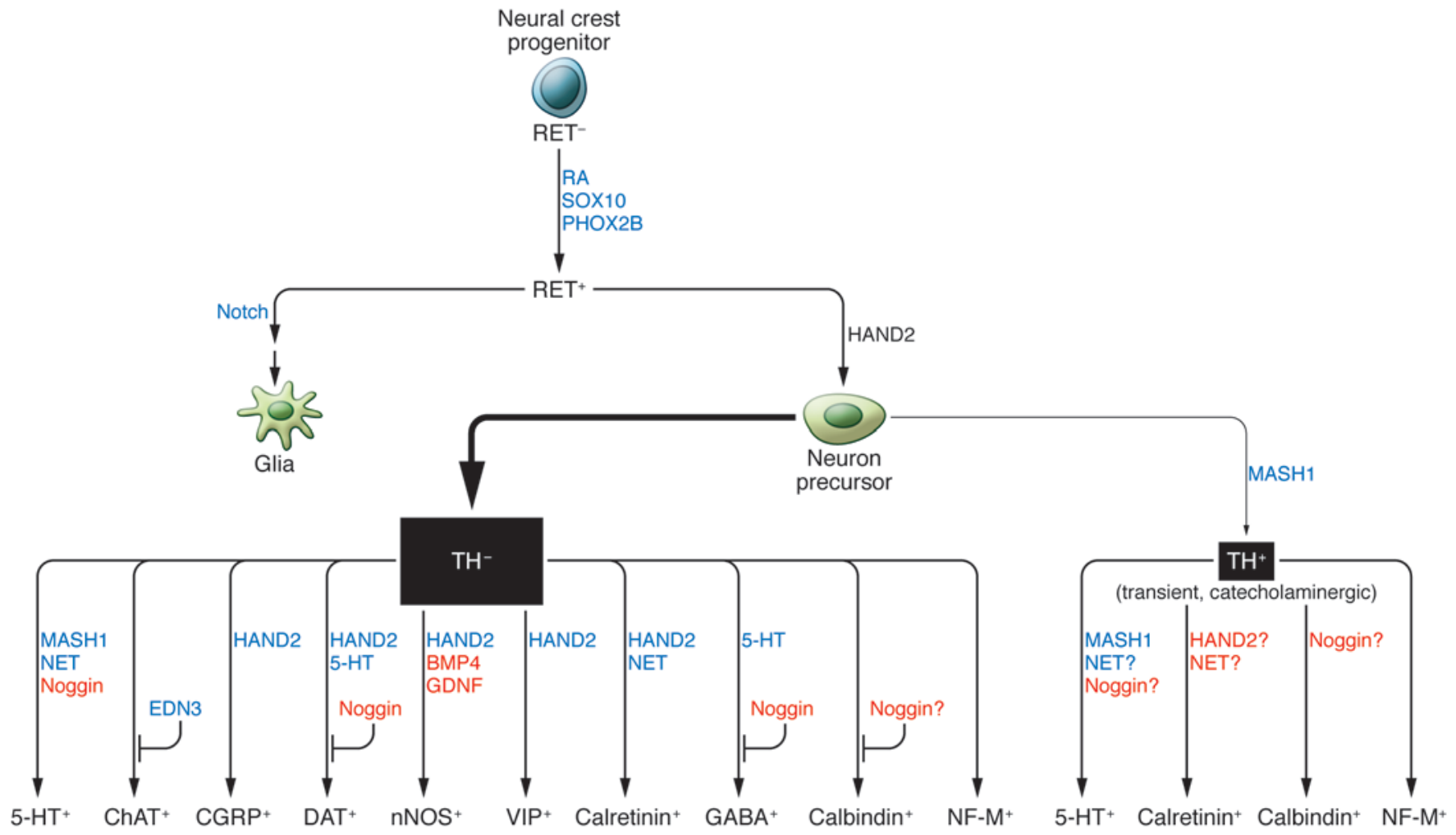

Figure 3. ENS precursor lineage relationships and neuronal subtype specification. Lineage relationships among enteric neuron subtypes remain poorly understood. This figure summarizes in vivo observations. Gain-of-function data are indicated in red. Loss-of-function data are indicated in blue. Most myenteric neurons arise from TH-negative precursors, as indicated by the relative thickness of arrows.

data. Figure 3 provides one model for enteric neuron subtype lineage relationships, reflecting recent data about transiently catecholaminergic (TC) cells. TC ENS precursors arise early in development (89) and absolutely require the transcription factor ASCL1/MASH1 (94). Serotonergic neurons, but not other subtypes, also require ASCL1/ MASH1 and were thought to arise exclusively from TC precursors. Fate-mapping studies of tyrosine hydroxylase-expressing cells now suggest that TC precursors give rise to only $30 \%$ of serotonergic neurons, but also can become calbindin-, calretinin-, and neurofilament-M-expressing neurons (95). Importantly, TC precursors give rise to less than $3 \%$ of myenteric neurons and to $13 \%$ of submucosal neurons in the mouse distal small intestine. It is unclear whether TC precursor-derived serotonergic neurons differ from non-TCderived serotonergic neurons. The complexity of these data highlights how little we understand enteric neuron subtype specification.

One additional critical question is how ratios of enteric neuron subtypes are controlled to produce integrated circuits and ganglia with diverse neuron types. If proliferating ENCDCs are lineage restricted early, establishment of ganglia with multiple neuron subtypes would require segregation and organization of committed precursors (96). An alternative mechanism that might support this outcome involves signals from one neuron affecting the phenotype of other neurons. In vivo data support this hypothesis, since loss of neuronal serotonin causes selective decreases in "late-born" dopaminergic and GABAergic neurons (97). Additionally, the norepinephrine reuptake transporter (NET) supports development of serotonergic and calretinin immunoreactive (IR) neurons (98). Neural activity also influences differentiation, as hindgut explants treated with tetrodotoxin or tetanus toxin have fewer nitrergic neurons but normal total neuron number (99). In contrast, depolarization of ENS precursors in culture increases tyrosine hydroxylase-expressing and vasoactive intestinal peptide-expressing (VIP-expressing) neurons but not nitrergic neurons (100). Signals from the ECM might also direct subtype differentiation. Culture of ENS precursors on collagen IV leads to more nNOS-IR neurons than growth on laminin or heparin sulfate (101). Mechanisms underlying this observation are not known.

Remarkably, enteric neuron subtype plasticity appears to continue after birth, at least in rodents, in which close proximity of the developing ENS to bowel lumen facilitates diet and intestinal microbe-induced changes in neuronal cell fate (102). In rats, the percentage of ChAT-IR neurons in colon myenteric plexus increases from P14 to P36, whereas the percentage of nNOS-IR neurons increases from $\mathrm{P} 1$ to $\mathrm{P} 5$ and then remains unchanged to P36 (103). These ratios can be altered by intraluminal lipids. Rats given daily colon enemas (at P7-P17) with the short-chain fatty acid butyrate had a larger percentage of myenteric and submucosal nNOS-IR and myenteric ChAT-IR neurons at P21 than do control rats (104). Butyrate-treated rats also had increased nitrergic and cholinergic neurotransmission and slowed colonic transit. Piglets whose mothers were fed n-3 polyunsaturated fatty acids during pregnancy and lactation also had increased ChAT-IR and decreased VIP-IR neurons in the jejunal submucosal plexus (105). These data suggest that maternal dietary factors can affect ENS subtype ratios in offspring during pregnancy or via altered breast milk composition. Thus, neuronal subtype specification appears 
to depend on a combination of intrinsic genetic programming and environmental factors. The duration of plasticity is not known but may underlie some human intestinal motility disorders and, if better understood, might suggest novel treatment strategies.

\section{Axon pathfinding and synaptogenesis: a tangled web of neurites}

Enteric neurons must extend processes to communicate with diverse cell types including other enteric neurons, interstitial cells of Cajal, smooth muscle, endothelial cells, mucosal epithelia, and intestinal glands (21). It is not clear whether subtype identity is established before enteric neurons innervate targets or what guides axons. This work is challenging because single enteric ganglia contain heterogeneous neuron subtypes and fasciculated fiber tracts have diverse axon types. Nonetheless, axons of different enteric neuron subtypes clearly have stereotypic projection patterns. For example, intracellular dye filling after electrophysiologic recording from guinea pig intrinsic primary afferent neurons (IPANs) shows projections circumferentially around bowel and then into mucosa (106). Studies using myotomy and myectomy followed by immunohistochemistry demonstrate that excitatory motor neuron axons project rostrally, while inhibitory motor neurons project caudally within the longitudinal axis of the myenteric plexus before entering circular or longitudinal muscle (106, 107). Elegant models of enteric neuron circuitry now exist based on decades of work $(1,22,108-110)$, but mechanisms needed to establish these connections remain obscure.

A few mouse mutations provide insight into axon guidance mechanisms. Mutations in the RET ligand neurturin or its preferred coreceptor GFR $\alpha 2$ reduce substance P-IR, but not VIP-IR, fibers innervating bowel circular muscle $(111,112)$. This phenotype could occur because of reduced neurite growth or branching, or might reflect problems with axon targeting to circular muscle. More convincing evidence that RET ligands direct neurite growth comes from mice that ectopically express GDNF from enteric glia. In these animals nitrergic, but not serotonergic or cholinergic fibers, redistribute toward the source of GDNF, suggesting a chemoattractive effect of GDNF on nitrergic axons (113). It is not known whether GDNF-dependent chemoattraction is direct or whether GDNF induces the production of new guidance receptors, rendering nitrergic neurons competent to respond to new attractant cues. Non-neurotrophic factors important for regulating ENS connectivity were also recently identified. Elegant analysis showed that mice with disruption of planar cell polarity genes Fzd3 and Celsr 3 have serious defects in axon pathfinding, but these were only seen with DiI or by expressing fluorescent proteins in small numbers of enteric neurons. These data highlight the utility of single-cell labeling techniques for identifying axon pathfinding defects in the ENS (114). Importantly, Celsr3 $3^{-/-}$mice have severe dysmotility, increased whole gut transit time, and disorganized colonic migrating motor complexes, but major neuronal subtypes are present and enteric neuron number is normal, suggesting that difficult-to-recognize innervation patterning defects may seriously affect bowel motility.

Following pathfinding, enteric neurons must form appropriate synaptic connections within the ENS. Although spontaneous neural activity and synaptic markers are present at E11.5 (115), elec- trophysiologic maturity is not reached until after birth, suggesting ongoing refinement of neural networks. Based on morphology and electrophysiology, mature enteric neurons are classified as Dogiel type I (DG I) or DG II (ref. 116 and Figure 2E). DG I neurons have a single axon, fast excitatory postsynaptic potentials, and somas with lamellar dendrites and include excitatory and inhibitory motor neurons (22). DG II neurons have multiple axon-like processes, prominent after-hyperpolarizing potentials, and smooth somas without lamellar dendrites and include IPANs. Although both DG I and DG II neurons are present by PO in mice, most DG I neurons possess filamentous instead of lamellar dendritic projections. The proportion of neurons with lamellar dendrites increases until adulthood (117). Furthermore, while the length of DG II circumferential projections remains constant despite dramatic increases in bowel circumference from PO to adulthood, DG I longitudinal axons continue to grow, though more slowly than does the bowel. In contrast, DG I neurons have mature electrophysiologic properties by P10, while DG II neuron electrophysiologic properties differ from adults at P10. These studies suggest that synaptic development, refinement, and remodeling occur postnatally in growing bowel and support the hypothesis that environmental disturbances in early life (infection, psychological stress, etc.) could profoundly influence adult bowel motility (118) by altering enteric neuron anatomy or subtype specification.

\section{Clinical implications}

Mechanisms controlling ENS development and function are complicated. Hundreds of genes control precursor survival, proliferation, migration, differentiation, subtype specification, polarity, neurite growth, axon pathfinding, and synaptogenesis. Tremendous basic research progress provides new insight, but advances have had little impact on care provided for people with motility disorders. Actionable ideas follow naturally from currently available data:

1. Vitamin A deficiency, a common global problem (119), might be a preventable cause of some birth defects including HSCR. Human studies in high-risk populations are needed to test this hypothesis. Note: Vitamin A excess also causes birth defects (120), so high doses must be avoided in pregnancy.

2. Some medicines cause HSCR-like disease in mice (20). Human epidemiologic studies identifying nongenetic risks for HSCR may lead to new prevention strategies.

3. Current clinical evaluation of the ENS is often limited to "present" or "absent," even when manometry suggests enteric neuropathy underlies life-threatening dysmotility. Evaluation is needed of neuronal subtypes, as well as tracking of neurites in biopsies from people with bowel motility disorders. New 3D visualization approaches make this possible $(121,122)$. Age- and region-specific control data are also needed, in order to know what ENS anatomy should be considered normal (123).

4. Next-generation genetic analysis should be incorporated into clinical testing for people with severe motility disorders. This will permit development of novel, individually targeted therapies.

5. Postnatal gut-derived neural stem cells can now be harvested from humans, cultured, and transplanted into animal models, where they partially restore function or improve outcome (124-129). This suggests that autotransplantation and gene editing 
might be used to treat bowel motility disorders and perhaps other serious medical problems. More work is needed to define how to grow and direct differentiation of these stem cells, although recent studies suggest that the gut environment might induce differentiation of many types of functioning enteric neurons and that these stem cells can integrate into existing ENS circuitry (130).

We remain optimistic that as mechanisms controlling bowel motility are better defined, new approaches to treatment, prevention, diagnosis, and cure will be developed to help people with serious bowel motility disorders.

\section{Acknowledgments}

We thank Rajarshi Sengupta for helpful comments on the manuscript. R.O. Heuckeroth is supported by the Irma and Nor- man Braman Endowment, The Children's Hospital of Philadelphia Research Institute, the Children's Discovery Institute of Washington University and St. Louis Children's Hospital (grants MD-II-2013-269 and CH-II-2010-390), the NIH (grant RO1 DK087715), and the Burroughs Wellcome Fund through a Clinical Scientist Award in Translational Research (award 1008525). E. Merrick Schill is supported in part by NIH grant F30 DK100101.

Address correspondence to: Robert O. Heuckeroth, Children's Hospital of Philadelphia Research Institute, 3615 Civic Center Boulevard, Abramson Research Center, Suite 1116i, Philadelphia, Pennsylvania 19104-4318, USA. Phone: 215.590.1209; E-mail: heuckerothr@email.chop.edu.
1. Furness JB. The enteric nervous system and neurogastroenterology. Nat Rev Gastroenterol Hepatol. 2012;9(5):286-294.

2. Heanue TA, Pachnis V. Enteric nervous system development and Hirschsprung's disease: advances in genetic and stem cell studies. Nat Rev Neurosci. 2007;8(6):466-479.

3. Wood JD. Taming the irritable bowel. Curr Pharm Des. 2013;19(1):142-156.

4. Heuckeroth RO. Hirschsprung disease. In: Faure C, DiLorenzo C, Thapar N, eds. Pediatric Neurogastroenterology: Gastrointestinal Motility And Functional Disorders In Children. New York, New York, USA: Springer; 2013:271-283.

5. Haricharan RN, Georgeson KE. Hirschsprung disease. Semin Pediatr Surg. 2008;17(4):266-275.

6. Lyford G, Foxx-Orenstein A. Chronic Intestinal Pseudoobstruction. Curr Treat Options Gastroenterol. 2004;7(4):317-325.

7. Tang DM, Friedenberg FK. Gastroparesis: approach, diagnostic evaluation, and management. Dis Mon. 2011;57(2):74-101.

8. Pfeiffer RF. Gastrointestinal dysfunction in Parkinson's disease. Parkinsonism Relat Disord. 2011;17(1):10-15.

9. Bagyanszki M, Bodi N. Diabetes-related alterations in the enteric nervous system and its microenvironment. World J Diabetes. 2012;3(5):80-93.

10. Moynes DM, Lucas GH, Beyak MJ, Lomax AE. Effects of inflammation on the innervation of the colon. Toxicol Pathol. 2014;42(1):111-117.

11. Brun P, et al. Toll-like receptor 2 regulates intestinal inflammation by controlling integrity of the enteric nervous system. Gastroenterology. 2013;145(6):1323-1333.

12. Margolis KG, et al. Enteric neuronal density contributes to the severity of intestinal inflammation. Gastroenterology. 2011;141(2):588-598.

13. Clairembault T, Leclair-Visonneau L, Neunlist M, Derkinderen P. Enteric glial cells: New players in Parkinson's disease? [published online ahead of print August 7, 2014]. Mov Disord. doi:10.1002/mds.25979.

14. Grathwohl SA, Steiner JA, Britschgi M, Brundin P. Mind the gut: secretion of $\alpha$-synuclein by enteric neurons. J Neurochem. 2013;125(4):487-490.

15. Hansen C, Li JY. Beyond $\alpha$-synuclein transfer: pathology propagation in Parkinson's disease. Trends Mol Med. 2012;18(5):248-255.
16. Obermayr F, Hotta R, Enomoto H, Young HM. Development and developmental disorders of the enteric nervous system. Nat Rev Gastroenterol Hepatol. 2013;10(1):43-57.

17. Simkin JE, Zhang D, Rollo BN, Newgreen DF. Retinoic acid upregulates ret and induces chain migration and population expansion in vagal neural crest cells to colonise the embryonic gut. PLoS One. 2013;8(5):e64077.

18. Burns AJ, Le Douarin NM. Enteric nervous system development: analysis of the selective developmental potentialities of vagal and sacral neural crest cells using quail-chick chimeras. Anat Rec. 2001;262(1):16-28.

19. Landman KA, Simpson MJ, Newgreen DF. Mathematical and experimental insights into the development of the enteric nervous system and Hirschsprung's disease. Dev Growth Differ. 2007;49(4):277-286.

20. Lake JI, Tusheva OA, Graham BL, Heuckeroth RO. Hirschsprung-like disease is exacerbated by reduced de novo GMP synthesis. JClin Invest. 2013;123(11):4875-4887.

21. Furness JB. Types of neurons in the enteric nervous system. J Auton Nerv Syst. 2000;81(1-3):87-96.

22. Furness JB. The Enteric Nervous System. Malden, Massachusetts, USA: Blackwell Publishing; 2006.

23. Lake JI, Heuckeroth RO. Enteric nervous system development: migration, differentiation, and disease. Am J Physiol Gastrointest Liver Physiol. 2013;305(1):G1-G24.

24. Sasselli V, Pachnis V, Burns AJ. The enteric nervous system. Dev Biol. 2012;366(1):64-73.

25. Newgreen DF, Dufour S, Howard MJ, Landman KA. Simple rules for a "simple" nervous system? Molecular and biomathematical approaches to enteric nervous system formation and malformation. Dev Biol. 2013;382(1):305-319.

26. Burns AJ, Thapar N. Advances in ontogeny of the enteric nervous system. Neurogastroenterol Motil. 2006;18(10):876-887

27. Heuckeroth RO, Lampe PA, Johnson EM, Milbrandt J. Neurturin and GDNF promote proliferation and survival of enteric neuron and glial progenitors in vitro. Dev Biol. 1998;200(1):116-129.

28. Uesaka T, Enomoto H. Neural precursor death is central to the pathogenesis of intestinal aganglionosis in Ret hypomorphic mice. J Neurosci. 2010;30(15):5211-5218.
29. Natarajan D, Marcos-Gutierrez C, Pachnis V, De Graaff E. Requirement of signalling by receptor tyrosine kinase RET for the directed migration of enteric nervous system progenitor cells during mammalian embryogenesis. Development. 2002;129(22):5151-5160.

30. Focke PJ, Schiltz CA, Jones SE, Watters JJ, Epstein ML. Enteric neuroblasts require the phosphatidylinositol 3-kinase pathway for GDNF-stimulated proliferation. J Neurobiol. 2001;47(4):306-317.

31. Chalazonitis A, Rothman TP, Chen J, Gershon MD. Age-dependent differences in the effects of GDNF and NT-3 on the development of neurons and glia from neural crest-derived precursors immunoselected from the fetal rat gut: expression of GFR $\alpha-1$ in vitro and in vivo. Dev Biol. 1998;204(2):385-406.

32. Wang $\mathrm{H}$, et al. The timing and location of glial cell line-derived neurotrophic factor expression determine enteric nervous system structure and function. J Neurosci. 2010;30(4):1523-1538.

33. Gianino S, Grider JR, Cresswell J, Enomoto H, Heuckeroth RO. GDNF availability determines enteric neuron number by controlling precursor proliferation. Development. 2003;130(10):2187-2198.

34. Schuchardt A, D'Agati V, Larsson-Blomberg L, Costantini F, Pachnis V. Defects in the kidney and enteric nervous system of mice lacking the tyrosine kinase receptor Ret. Nature. 1994;367(6461):380-383.

35. Shimotake T, Go S, Inoue K, Tomiyama H, Iwai N. A homozygous missense mutation in the tyrosine E kinase domain of the RET proto-oncogene in an infant with total intestinal aganglionosis. Am J Gastroenterology. 2001;96(4):1286-1291.

36. Niederreither K, Vermot J, Le Roux I, Schuhbaur B, Chambon P, Dolle P. The regional pattern of retinoic acid synthesis by RALDH2 is essential for the development of posterior pharyngeal arches and the enteric nervous system. Development. 2003;130(11):2525-2534.

37. Airaksinen MS, Saarma M. The GDNF family: signalling, biological functions and therapeutic value. Nat Rev Neurosci. 2002;3(5):383-394.

38. Baloh RH, Enomoto H, Johnson EMJ, Milbrandt J. The GDNF family ligands and receptors implications for neural development. Curr Opin Neurobiol. 2000;10(1):103-110.

39. Enomoto H, et al. GFR $\alpha 1$-deficient mice have 
deficits in the enteric nervous system and kidneys. Neuron. 1998;21(2):317-324.

40. Enomoto H, et al. GFR $\alpha 1$ expression in cells lacking RET is dispensable for organogenesis and nerve regeneration. Neuron. 2004;44(4):623-636.

41. Sanchez MP, Silos-Santiago I, Frisen J, He B, Lira SA, Barbacid M. Renal agenesis and the absence of enteric neurons in mice lacking GDNF. Nature. 1996;382(6586):70-73.

42. Pichel JG, et al. Defects in enteric innervation and kidney development in mice lacking GDNF. Nature. 1996;382(6586):73-76.

43. Moore MW, et al. Renal and neuronal abnormalities in mice lacking GDNF. Nature. 1996;382(6586):76-79.

44. Cacalano G, et al. GFRa1 is an essential receptor component for GDNF in the developing nervous system and kidney. Neuron. 1998;21(1):53-62.

45. Amiel J, et al. Hirschsprung disease, associated syndromes and genetics: a review. J Med Genet. 2008;45(1):1-14.

46. Emison ES, et al. A common sex-dependent mutation in a RET enhancer underlies Hirschsprung disease risk. Nature. 2005;434(7035):857-863.

47. Nishiyama C, et al. Trans-mesenteric neural crest cells are the principal source of the colonic enteric nervous system. Nat Neurosci. 2012;15(9):1211-1218.

48. Young HM, et al. Colonizing while migrating: how do individual enteric neural crest cells behave? BMC Biol. 2014;12:23.

49. Young HM, Jones BR, McKeown SJ. The projections of early enteric neurons are influenced by the direction of neural crest cell migration. J Neurosci. 2002;22(14):6005-6018.

50. Barlow AJ, Wallace AS, Thapar N, Burns AJ. Critical numbers of neural crest cells are required in the pathways from the neural tube to the foregut to ensure complete enteric nervous system formation. Development. 2008;135(9):1681-1691.

51. Burns AJ, Champeval D, Le Douarin NM. Sacral neural crest cells colonise aganglionic hindgut in vivo but fail to compensate for lack of enteric ganglia. Dev Biol. 2000;219(1):30-43.

52. Wu JJ, Chen JX, Rothman TP, Gershon MD. Inhibition of in vitro enteric neuronal development by endothelin-3: mediation by endothelin B receptors. Development. 1999;126(6):1161-1173.

53. Nagy N, Goldstein AM. Endothelin-3 regulates neural crest cell proliferation and differentiation in the hindgut enteric nervous system. Dev Biol. 2006;293(1):203-217.

54. Barlow A, de Graaff E, Pachnis V. Enteric nervous system progenitors are coordinately controlled by the $G$ protein-coupled receptor EDNRB and the receptor tyrosine kinase RET. Neuron. 2003;40(5):905-916.

55. Hosoda K, et al. Targeted and natural (piebaldlethal) mutations of endothelin-B receptor gene produce megacolon associated with spotted coat color in mice. Cell. 1994;79(7):1267-1276.

56. Puffenberger EG, et al. A missense mutation of the endothelin-B receptor gene in multigenic Hirschsprung's disease. Cell. 1994;79(7):1257-1266.

57. Kapur RP, Sweetser DA, Doggett B, Siebert JR, Palmiter RD. Intercellular signals downstream of endothelin receptor-B mediate colonization of the large intestine by enteric neuroblasts. Development. 1995;121(11):3787-3795.

58. Kapur RP, Yost C, Palmiter RD. Aggregation chimeras demonstrate that the primary defect responsible for aganglionic megacolon in lethal spotted mice is not neuroblast autonomous. Development.1993;117(3):993-999.

59. Sukegawa A, et al. The concentric structure of the developing gut is regulated by Sonic hedgehog derived from endodermal epithelium. Development. 2000;127(9):1971-1980.

60 . Young HM, et al. Dynamics of neural crest-derived cell migration in the embryonic mouse gut. Dev Biol. 2004;270(2):455-473.

61. Druckenbrod NR, Epstein ML. The pattern of neural crest advance in the cecum and colon. Dev Biol. 2005;287(1):125-133.

62. Anderson RB, Turner KN, Nikonenko AG, Hemperly J, Schachner M, Young HM. The cell adhesion molecule 11 is required for chain migration of neural crest cells in the developing mouse gut. Gastroenterology. 2006;130(4):1221-1232.

63. Okamoto N, et al. Hydrocephalus and Hirschsprung's disease with a mutation of L1CAM. J Hum Genet. 2004;49(6):334-337.

64. Parisi MA, et al. Hydrocephalus and intestinal aganglionosis: is L1CAM a modifier gene in Hirschsprung disease? Am JMed Genet. 2002;108(1):51-56.

65. Akbareian SE, et al. Enteric neural crest-derived cells promote their migration by modifying their microenvironment through tenascin- $\mathrm{C}$ production. Dev Biol. 2013;382(2):446-456.

66. Anderson RB. Matrix metalloproteinase- 2 is involved in the migration and network formation of enteric neural crest-derived cells. Int J Dev Biol. 2010;54(1):63-69.

67. Burns AJ, Douarin NM. The sacral neural crest contributes neurons and glia to the post-umbilical gut: spatiotemporal analysis of the development of the enteric nervous system. Development. 1998;125(21):4335-4347.

68. Goto A, et al. GDNF and endothelin 3 regulate migration of enteric neural crest-derived cells via protein kinase A and Rac1. J Neurosci. 2013;33(11):4901-4912.

69. Ridley AJ, et al. Cell migration: integrating signals from front to back. Science. 2003;302(5651):1704-1709.

70. Lim CJ, et al. Integrin-mediated protein kinase A activation at the leading edge of migrating cells. Mol Biol Cell. 2008;19(11):4930-4941.

71. Asai N, et al. Targeted mutation of serine 697 in the Ret tyrosine kinase causes migration defect of enteric neural crest cells. Development. 2006;133(22):4507-4516.

72. Vohra BP, Fu M, Heuckeroth RO. Protein kinase Czeta and glycogen synthase kinase- $3 \beta$ control neuronal polarity in developing rodent enteric neurons, whereas SMAD specific E3 ubiquitin protein ligase 1 promotes neurite growth but does not influence polarity. J Neurosci. 2007;27(35):9458-9468.

73. Fukuda T, Kiuchi K, Takahashi M. Novel mechanism of regulation of Rac activity and lamellipodia formation by RET tyrosine kinase. J Biol Chem. 2002;277(21):19114-19121.

74. Fu M, et al. Vitamin A facilitates enteric nervous system precursor migration by reducing Pten accumulation. Development. 2010;137(4):631-640.

75. Hall A. Rho GTPases and the control of cell behaviour. Biochem Soc Trans. 2005; 33(pt 5):891-895.

76. Stewart AL, Young HM, Popoff M, Anderson RB. Effects of pharmacological inhibition of small GTPases on axon extension and migration of enteric neural crest-derived cells. Dev Biol. 2007;307(1):92-104.

77. Zhang Y, Kim TH, Niswander L. Phactr4 regulates directional migration of enteric neural crest through PP1, integrin signaling, and cofilin activity. Genes Dev. 2012;26(1):69-81.

78. Sato Y, Heuckeroth RO. Retinoic acid regulates murine enteric nervous system precursor proliferation, enhances neuronal precursor differentiation, and reduces neurite growth in vitro. $D e v$ Biol. 2008;320(1):185-198.

79. Jiang Y, Liu MT, Gershon MD. Netrins and DCC in the guidance of migrating neural crest-derived cells in the developing bowel and pancreas. Dev Biol. 2003;258(2):364-384.

80. Uesaka T, Nagashimada M, Enomoto H. GDNF signaling levels control migration and neuronal differentiation of enteric ganglion precursors. J Neurosci. 2013;33(41):16372-16382.

81. Hackett-Jones EJ, Landman KA, Newgreen DF, Zhang D. On the role of differential adhesion in gangliogenesis in the enteric nervous system. J Theor Biol. 2011;287:148-159.

82. Fu M, Vohra BP, Wind D, Heuckeroth RO. BMP signaling regulates murine enteric nervous system precursor migration, neurite fasciculation, and patterning via altered Ncam1 polysialic acid addition. Dev Biol. 2006;299(1):137-150.

83. Faure C, et al. Gangliogenesis in the enteric nervous system: roles of the polysialylation of the neural cell adhesion molecule and its regulation by bone morphogenetic protein-4. Dev Dyn. 2007;236(1):44-59.

84. Breau MA, et al. Lack of beta1 integrins in enteric neural crest cells leads to a Hirschsprung-like phenotype. Development. 2006;133(9):1725-1734.

85. Breau MA, Dahmani A, Broders-Bondon F, Thiery JP, Dufour S. $\beta 1$ integrins are required for the invasion of the caecum and proximal hindgut by enteric neural crest cells. Development. 2009;136(16):2791-2801.

86. Lei J, Howard MJ. Targeted deletion of Hand2 in enteric neural precursor cells affects its functions in neurogenesis, neurotransmitter specification and gangliogenesis, causing functional aganglionosis. Development. 2011;138(21):4789-4800.

87. D'Autreaux F, Morikawa Y, Cserjesi P, Gershon MD. Hand2 is necessary for terminal differentiation of enteric neurons from crest-derived precursors but not for their migration into the gut or for formation of glia. Development. 2007;134(12):2237-2249.

88. D'Autreaux F, et al. Expression level of Hand2 affects specification of enteric neurons and gastrointestinal function in mice. Gastroenterology. 2011;141(2):576-587.

89. Pham TD, Gershon MD, Rothman TP. Time of origin of neurons in the murine enteric nervous system: sequence in relation to phenotype. J Comp Neurol. 1991;314(4):789-798.

90. Chalazonitis A, et al. Bone morphogenetic pro- 
tein regulation of enteric neuronal phenotypic diversity: relationship to timing of cell cycle exit. JComp Neurol. 2008;509(5):474-492.

91. Bergner AJ, et al. Birthdating of myenteric neuron subtypes in the small intestine of the mouse. JComp Neurol. 2014;522(3):514-527.

92. Chalazonitis A, Kessler JA. Pleiotropic effects of the bone morphogenetic proteins on development of the enteric nervous system. Dev Neurobiol. 2012;72(6):843-856.

93. Yan $\mathrm{H}$, Bergner AJ, Enomoto H, Milbrandt J, Newgreen DF, Young HM. Neural cells in the esophagus respond to glial cell line-derived neurotrophic factor and neurturin, and are RETdependent. Dev Biol. 2004;272(1):118-133.

94. Blaugrund E, et al. Distinct subpopulations of enteric neuronal progenitors defined by time of development, sympathoadrenal lineage markers, and Mash-1-dependence. Development. 1996;122(1):309-320.

95. Obermayr F, Stamp LA, Anderson CR, Young HM. Genetic fate-mapping of tyrosine hydroxylaseexpressing cells in the enteric nervous system. Neurogastroenterol Motil. 2013;25(4):e283-e291.

96. Cheeseman BL, Zhang D, Binder BJ, Newgreen DF, Landman KA. Cell lineage tracing in the developing enteric nervous system: superstars revealed by experiment and simulation. $J R$ Soc Interface. 2014;11(93):20130815.

97. Li Z, et al. Essential roles of enteric neuronal serotonin in gastrointestinal motility and the development/survival of enteric dopaminergic neurons. J Neurosci. 2011;31(24):8998-9009.

98. Li Z, Caron MG, Blakely RD, Margolis KG, Gershon MD. Dependence of serotonergic and other nonadrenergic enteric neurons on norepinephrine transporter expression. J Neurosci. 2010;30(49):16730-16740.

99. Hao MM, et al. The role of neural activity in the migration and differentiation of enteric neuron precursors. Neurogastroenterol Motil. 2010;22(5):e127-e137.

100.Chevalier J, et al. Activity-dependent regulation of tyrosine hydroxylase expression in the enteric nervous system. J Physiol. 2008;586(7):1963-1975.

101. Raghavan S, Bitar KN. The influence of extracellular matrix composition on the differentiation of neuronal subtypes in tissue engineered innervated intestinal smooth muscle sheets. Biomaterials. 2014;35(26):7429-7440.

102. Neunlist M, Schemann M. Nutrient-induced changes in the phenotype and function of the enteric nervous system. J Physiol. 2014; 592(pt 14):2959-2965.

103.de Vries P, Soret R, Suply E, Heloury Y, Neunlist M. Postnatal development of myenteric neurochemical phenotype and impact on neuromuscular transmission in the rat colon. Am JPhysiol Gastrointest Liver Physiol. 2010;299(2):G539-G547.

104. Suply E, de Vries P, Soret R, Cossais F, Neunlist M.
Butyrate enemas enhance both cholinergic and nitrergic phenotype of myenteric neurons and neuromuscular transmission in newborn rat colon. Am J Physiol Gastrointest Liver Physiol. 2012;302(12):G1373-G1380.

105. De Quelen F, Chevalier J, Rolli-Derkinderen M, Mourot J, Neunlist M, Boudry G. n-3 polyunsaturated fatty acids in the maternal diet modify the postnatal development of nervous regulation of intestinal permeability in piglets. J Physiol. 2011;589(pt 17):4341-4352.

106. Messenger JP, Furness JB. Projections of chemically-specified neurons in the guinea-pig colon. Arch Histol Cytol. 1990;5(53):467-495.

107. Sang Q, Williamson S, Young HM. Projections of chemically identified myenteric neurons of the small and large intestine of the mouse. J Anat. 1997;190(pt 2):209-222.

108. Wood JD. Enteric nervous system: reflexes, pattern generators and motility. Curr Opin Gastroenterol. 2008;24(2):149-158.

109. Bornstein JC, Costa M, Grider JR. Enteric motor and interneuronal circuits controlling motility. Neurogastroenterol Motil. 2004;16(suppl 1):34-38.

110.Schemann M, Neunlist M. The human enteric nervous system. Neurogastroenterol Motil. 2004;16(suppl 1):55-59.

111. Heuckeroth RO, et al. Gene targeting reveals a critical role for neurturin in the development and maintenance of enteric, sensory, and parasympathetic neurons. Neuron. 1999;22(2):253-263.

112. Rossi J, et al. Retarded growth and deficits in the enteric and parasympathetic nervous system in mice lacking GFR $\alpha 2$, a functional neurturin receptor. Neuron. 1999;22(2):243-252.

113. Wang $\mathrm{H}$, et al. The timing and location of glial cell line-derived neurotrophic factor expression determine enteric nervous system structure and function. JNeurosci. 2010;30(4):1523-1538.

114. Sasselli V, Boesmans W, Berghe PV, Tissir F, Goffinet AM, Pachnis V. Planar cell polarity genes control the connectivity of enteric neurons. JClin Invest. 2013;123(4):1763-1772.

115. Hao MM, et al. Early emergence of neural activity in the developing mouse enteric nervous system. J Neurosci. 2011;31(43):15352-15361.

116. Dogiel AS. Ueber den bau der ganglien in den geflechten des darmes und der gallenblase des menschen und der saugethiere. Arch Anat Physiol Leip Anat Abt Jg. 1899;1899:130-158.

117. Foong JPP, Nguyen TV, Furness JB, Bornstein JC, Young HM. Myenteric neurons of the mouse small intestine undergo significant electrophysiological and morphological changes during postnatal development.JPhysiol. 2012;590(pt 10):2375-2390.

118. Bradford K, et al. Association between early adverse life events and irritable bowel syndrome. Clin Gastroenterol Hepatol. 2012;10(4):385-390.

119. West KP Jr. Extent of vitamin A deficiency among preschool children and women of reproductive age. J Nutr. 2002;132(9 suppl):2857S-2866S

120. Geelen JAG. Hypervitaminosis A induced teratogenesis. CRC Crit Rev Toxicol. 1979;6(4):351-375.

121. Liu YA, et al. 3-D imaging, illustration, and quantitation of enteric glial network in transparent human colon mucosa. Neurogastroenterol Motil. 2013;25(5):e324-e338.

122.Fu YY, Peng SJ, Lin HY, Pasricha PJ, Tang SC. 3-D imaging and illustration of mouse intestinal neurovascular complex. Am J Physiol Gastrointest Liver Physiol. 2013;304(1):G1-G11.

123. Knowles $\mathrm{CH}$, et al. The London Classification of gastrointestinal neuromuscular pathology: report on behalf of the Gastro 2009 International Working Group. Gut. 2010;59(7):882-887.

124. Burns AJ, Thapar N. Neural stem cell therapies for enteric nervous system disorders. Nat Rev Gastroenterol Hepatol. 2013;11(5):317-328.

125. Hotta R, Thapar N. Advances in enteric neurobiology: how close are we to clinical use? J Pediatr Gastroenterol Nutr. 2011;53(suppl 2):S43-S45.

126. Hotta R, Natarajan D, Burns AJ, Thapar N. Stem cells for GI motility disorders. Curr Opin Pharmacol. 2011;11(6):617-623.

127. Metzger M, Caldwell C, Barlow AJ, Burns AJ, Thapar N. Enteric nervous system stem cells derived from human gut mucosa for the treatment of aganglionic gut disorders. Gastroenterology. 2009;136(7):2214-2225.

128. Theocharatos S, Wilkinson DJ, Darling S, Wilm B, Kenny SE, Edgar D. Regulation of progenitor cell proliferation and neuronal differentiation in enteric nervous system neurospheres. PLoS One. 2013;8(1):e54809.

129. Wilkinson DJ, Edgar DH, Kenny SE. Future therapies for Hirschsprung's disease. Semin Pediatr Surg. 2012;21(4):364-370.

130. Hotta R, et al. Transplanted progenitors generate functional enteric neurons in the postnatal colon. J Clin Invest. 2013;123(3):1182-1191.

131. Chalazonitis A, et al. Neurotrophin-3 is required for the survival-differentiation of subsets of developing enteric neurons. J Neurosci. 2001;21(15):5620-5636.

132. Zaitoun I, et al. Altered neuronal density and neurotransmitter expression in the ganglionated region of Ednrb null mice: implications for Hirschsprung's disease. Neurogastroenterol Motil. 2013;25(3):e233-e244.

133. Sandgren K, Larsson LT, Ekblad E. Widespread changes in neurotransmitter expression and number of enteric neurons and interstitial cells of Cajal in lethal spotted mice: an explanation for persisting dysmotility after operation for Hirschsprung's disease? Dig Dis Sci. 2002;47(5):1049-1064

134.Sang Q, Ciampoli D, Greferath U, Sommer L, Young HM. Innervation of the esophagus in mice that lack MASH1. J Comp Neurol. 1999;408(1):1-10. 